\title{
Influência da prática de atividade física sobre a qualidade de vida de usuários do SUS
}

\author{
The influence of the practice of physical activity on the quality of life \\ of SUS's patients
}

Joel Saraiva Ferreira', Sandra Helena Correia Diettrich ${ }^{2}$, Danielly Amado Pedro ${ }^{\mathbf{3}}$

RESUMO O estudo objetivou verificar a influência da atividade física sobre a qualidade de vida de participantes e não participantes de um Programa de Atividade Física, atendidos nas Unidades Básicas de Saúde da Família, no município de Campo Grande (MS). A população foi constituída de 90 indivíduos com mais de 40 anos, de ambos os sexos, os quais responderam ao questionário SF-36. Os resultados demonstraram diferença estatisticamente significativa entre os grupos, com valores melhores a favor da população praticante de atividade física. Foi possível concluir que tal prática pode contribuir para a melhora da qualidade de vida em programas de promoção da saúde, como o Estratégia Saúde da Família.

PALAVRAS-CHAVE Qualidade de vida; Promoção da saúde; Atividade física; Saúde da família.

ABSTRACT The study aimed to investigate the influence of physical activity on the quality of life of participants and not participants of a Physical Activity Program attended in the Family Health Basic Units, in the municipality of Campo Grande-MS. The population was composed of 90 individuals over 40 years, of both sexes, who replied to the questionnaire SF-36. Results showed statistically significant differences between groups, with better values for the physical activity practitioner population. It was possible to conclude that such practice can contribute to the improvement of the quality of life in health promotion programs, such as the Family Health Strategy.

KEYWORDS Quality of life; Health promotion; Physical activity; Family health.

\footnotetext{
1 Universidade Federal de Mato Grosso do Sul (UFMS) - Campo Grande (MS), Brasil.

falecomjoel@hotmail.com

2 Universidade Federal de Mato Grosso do Sul (UFMS) - Campo Grande (MS), Brasil.

shcd1@hotmail.com

3 Universidade Federal de Mato Grosso do Sul (UFMS) - Campo Grande (MS), Brasil. daniellyamado@gmail.com
} 


\section{Introdução}

A expressão qualidade de vida, muito discutida nos dias atuais, engloba múltiplos aspectos da vida de um indivíduo e compreende diversos fatores, entre os quais estão as dimensões físicas e emocionais. Para Castellón e Pino (2003), a qualidade de vida é constituída por vários componentes, que podem ser agrupados em: a) bem-estar físico; b) bem-estar material; c) bem-estar social; d) desenvolvimento e atividade; e e) bem-estar emocional.

Conforme Forattini (1992), qualidade de vida é um conceito difícil de ser definido, sendo, por isso, muito mais difícil de ser medido. De acordo com o autor, para estimar a qualidade de vida, aventa-se o emprego de vários dados dos quais resultariam medidas que, de forma genérica, podem ser definidas como objetivas e subjetivas.

Por ser também de caráter subjetivo, a qualidade de vida apresenta-se de forma diferente de pessoa para pessoa, pois depende do entendimento que cada uma tem sobre o assunto e do sentido que dá à sua vida. Iglesias (2002) afirma que os indivíduos têm percepções particulares sobre seus objetivos e planos de vida, portanto, a cada um cabe julgar o quanto as suas expectativas foram alcançadas, a despeito de todos os problemas encontrados. Sendo assim, qualidade de vida pode ser compreendida como a percepção de bem-estar resultante de um conjunto de parâmetros individuais e socioambientais, modificáveis ou não, que caracterizam as condições em que vive o ser humano (LANDEIRO ET AL., 2011; MONTEIRO ET AL., 2010). Assim, a medida de qualidade de vida deve compreender a satisfação dos indivíduos com relação aos aspectos considerados importantes para esses mesmos indivíduos.

No que se refere à relação entre a qualidade de vida e a saúde, Nahas (2013) salienta que o ser humano busca indefinidamente a melhoria de suas condições de vida e o máximo de autonomia possível durante toda a sua existência. Múltiplos fatores têm permitido a humanidade progredir nesse sentido, sendo relevante a evolução das ciências médicas na área da saúde, as alterações do ambiente e a transformação positiva do estilo de vida.

O estilo de vida é um dos componentes do modelo epidemiológico de saúde pública, denominado Campo de Saúde (DEVER, 1988). O grau de acesso em cada conceito do Campo de Saúde é determinado pelo ambiente, pela biologia humana e pelos serviços de saúde, enquanto estilo de vida é uma forma de analisar a qualidade de vida.

No estilo de vida, entre outros comportamentos do ser humano, tem-se a prática da atividade física. A relação entre saúde e prática de atividade física é tida como positiva pelos pesquisadores da área. Estudos confirmam que essa prática atua na prevenção de doenças, assim como na manutenção e recuperação da saúde do indivíduo em todas as faixas etárias (PAFFENBARGER ET AL., 1996; BLAIR ET AL., 1996; PATE ET AL., 1995; MILES, 2007).

Um estilo de vida ativo também pode ser considerado um fator influenciador para a promoção da saúde e a melhoria da qualidade de vida, sendo o estilo de vida definido como um conjunto de ações habituais que refletem as atitudes, os valores e as oportunidades na vida das pessoas (TAIROVA; DI LORENZI, 2011). Um estilo de vida ativo, resultante da prática de atividade física, contribui para a boa condição física, sendo considerado por Araújo e Araújo (2000) um dos fatores importantes para a prevenção e o tratamento de doenças e para a manutenção da saúde, bem como um instrumento precioso para a melhoria de qualidade de vida das pessoas.

Pelas evidências quanto aos efeitos benéficos que produz, a atividade física vem sendo crescentemente inserida em programas de promoção de hábitos saudáveis de vida, de prevenção e mesmo de controle de doenças (MITNITSKI ET AL., 2005 APUD KAYSER ET AL., 2012), considerando que a prática de atividade física regular reduz o risco de mortes prematuras, doenças do coração, acidente vascular cerebral, câncer de cólon, mama e diabetes tipo II. Atua no controle de valores pressóricos 
e, consequentemente, da pressão arterial, previne o ganho de peso (diminuindo o risco de obesidade), auxilia na prevenção ou redução da osteoporose, promove bem-estar, reduz o estresse, a ansiedade e a depressão, entre outros (WHO, 2004).

A implantação e o apoio aos programas de atividades físicas vinculados às políticas públicas são uma das metas do Ministério da Saúde, no sentido de promover a saúde e a qualidade de vida da população (BRASIL, 2006), a partir da agenda de compromissos pela saúde, de 2005, do Ministério da Saúde, e pela aprovação da Política Nacional de Promoção da Saúde, de 2006. O Pacto pela Vida define a atividade física como uma das suas macroprioridades, que pretende, entre outros: enfatizar a mudança de comportamento da população de forma a internalizar a responsabilidade individual da prática de atividade física regular; articular e promover os diversos programas de promoção de atividade física já existentes e apoiar a criação de outros (BRASIL, 2006).

Para Malta et al. (2009), a indução de atividades físicas/práticas corporais reflete o reconhecimento da importância conferida a um modo de viver ativo como fator de proteção à saúde. Nesse contexto, é possível assumir que as Unidades Básicas de Saúde (UBS) seriam locais privilegiados para a promoção da saúde, mediante o oferecimento da prática regular de atividade física, tendo em vista que atenderiam parte da população que não possui acesso a esses programas de qualidade ou que pertence a minorias altamente suscetíveis a um estilo de vida inativo (KOKUBUN ET AL., 2007).

"Promover a qualidade de vida e reduzir vulnerabilidade e riscos à saúde relacionados aos seus determinantes e condicionantes modos de viver, condições de trabalho, habitação, ambiente, educação, lazer, cultura, acesso a bens e serviços essenciais" - é o objetivo da Política Nacional de Promoção da Saúde, implantada pelo Ministério da Saúde, e tem como propósito planejar, realizar, analisar e avaliar o trabalho em saúde. Tal documento propõe o desenvolvimento de ações ligadas a várias áreas, entre elas, práticas corporais/ atividade física (BRASIL, 2006B P. 17).

Com relação, especificamente, às práticas corporais/atividade física, propõe-se que sejam implantadas ações na rede básica de saúde e na comunidade relacionadas a aconselhamento/divulgação; intersetorialidade; mobilização de parceiros; monitoramento e avaliação (BRASIL, 2006).

Mediante as informações supracitadas, verifica-se que a prática de atividade física é um dos comportamentos que podem contribuir para a qualidade de vida dos indivíduos e/ou coletividades, bem como promover a saúde dos mesmos, quando realizados individualmente ou por meio de programas sustentados por políticas nacionais pactuadas nas diferentes esferas de governo.

No município de Campo Grande, capital do Mato Grosso do Sul, por meio das ações de profissionais de Educação Física, são oferecidos orientação e acompanhamento de atividades físicas aos usuários do Sistema Único de Saúde (SUS) que buscam atendimento junto às Unidades Básicas de Saúde da Família (UBSF). Nem todas as UBSF contam com esse serviço, e, mesmo naquelas onde ele é oferecido, há pacientes que preferem não participar das atividades.

Nas UBSF onde é oferecido o serviço de atividade física/práticas corporais, esse é organizado em grupos específicos, diferenciados conforme os ciclos de vida ou a condição física (hipertensos, diabéticos, gestantes, entre outros). Assim, as atividades oferecidas possibilitam ao usuário do SUS acessar um programa de promoção da saúde, usufruindo de seus benefícios, entre os quais, a possível melhoria da qualidade de vida.

Coutinho (2005) afirma que, no Brasil, já é possível identificar algumas iniciativas de Equipes de Saúde da Família desenvolvendo os projetos relacionados à prática de atividade física como forma de promoção da saúde ou prevenção de doenças. Putzel (2011) considera que tais iniciativas tornam viáveis 
a exploração do campo e a identificação das possíveis respostas dos usuários e profissionais da saúde a respeito dos efeitos da atividade física orientada no contexto do SUS.

Posto isso, o presente estudo objetivou verificar a influência da atividade física sobre a qualidade de vida de Participantes de um Programa de Atividade Física (PPAF) e de Não Participantes de um Programa de Atividade Física (NPPAF) que buscam atendimento nas UBSF de um dos distritos sanitários do município de Campo Grande (MS).

\section{Materiais e métodos}

O estudo se caracterizou como exploratório e comparativo, com delineamento transversal. Para caracterização sociodemográfica da população investigada, foi utilizado um questionário com o qual foram obtidos dados referentes a: idade, sexo e local de residência.

A população investigada foi constituída de indivíduos com mais de 40 anos de idade, de ambos os sexos, residentes no Distrito Sul do município de Campo Grande (MS), atendidos pelas UBSF daquela região, compondo um grupo de 90 pessoas, sendo que, dessas, 45 eram PPAF e 45 eram NPPAF. Para selecionar a amostra, foi aplicada a equação estatística proposta por Krejcie e Morgan (1970), considerando, para isso, a população adscrita no distrito sanitário investigado.

Os dados foram coletados por meio da aplicação do questionário SF-36 (já traduzido e validado para a língua portuguesa), o qual é um instrumento genérico de avaliação de qualidade de vida, multidimensional, formado por 36 itens englobados em 8 escalas ou componentes: capacidade funcional, aspectos físicos, dor, estado geral da saúde, vitalidade, aspectos sociais, aspectos emocionais e saúde mental. As pontuações dos escores de qualidade de vida classificadas nesse instrumento vão de 0 (zero), que é a pior condição, até 100 (cem), que é a melhor condição possível (CICONELLI ET AL., 1999).

Para ambos os grupos, a aplicação do instrumento de coleta de dados foi feita de forma individualizada, em uma sala na UBSF disponibilizada para esse fim, de modo a preservar a privacidade do indivíduo e não atrapalhar as atividades dos demais profissionais de saúde. O questionário foi apresentado aos participantes e preenchido pelos componentes da equipe de pesquisa.

As análises estatísticas foram realizadas com o auxílio do programa estatístico BioEstat 5.3, com o qual se aplicou a estatística descritiva para caracterizar cada um dos subgrupos (PPAF ou NPPAF), além dos testes que demonstraram as diferenças entre os escores obtidos com o questionário SF-36. Para essa comparação, utilizou-se o teste de Mann-Whitney, pois uma análise anterior demonstrou que os dados tinham comportamento não paramétrico. Nesse caso, adotou-se um nível de significância de 5\%.

Os escores obtidos com a aplicação do questionário SF-36 foram organizados em tabelas, distribuídos por quartil (quatro grupos de $25 \%$ cada um deles) obtido em cada grupo da pesquisa. Com isso, foram formadas quatro classes: 1) Valores muito baixos: menores que o percentil 25 da amostra; 2) Valores baixos: do percentil 25até o percentil 49 da amostra; 3) Valores elevados: do percentil50 até o percentil 74 da amostra; 4) Valores muito elevados: superiores ou iguais ao percentil $75 \mathrm{da}$ amostra.

Todos os indivíduos pesquisados foram convidados a fazer parte do estudo, e os dados só foram coletados após concordância e assinatura do Termo de Consentimento Livre e Esclarecido (TCLE). A presente pesquisa foi aprovada pelo Comitê de Ética em Pesquisa com Seres Humanos da Universidade Federal de Mato Grosso do Sul (UFMS), protocolo n. ${ }^{\circ} 1181$. 


\section{Apresentação e discussão dos resultados}

Os dados obtidos no estudo são apresentados a seguir. Na tabela 1, estão expressos os valores descritivos da amostra do grupo PPAF e do grupo NPPAF, delimitados para este estudo, distribuídos conforme o sexo e a faixa etária.
Na tabela 2, os dados apresentados referem-se aos escores de cada domínio avaliado com o questionário SF-36, distribuídos em quatro classes conforme os valores percentilares $25 \%$, $50 \%$ e $75 \%$, nos grupos PPAF e NPPAF.

Por fim, a tabela 3 apresenta uma comparação entre os valores obtidos em cada domínio do questionário SF-36, comparando os dois grupos.

Tabela 1. Caracterização dos grupos PPEF e NPPEF, realizados em Unidades Básicas de Saúde da Família de Campo Grande (MS), conforme sexo e faixa etária $(n=90)$

\begin{tabular}{|c|c|c|c|c|c|c|c|}
\hline \multirow[t]{2}{*}{ Variável } & & \multicolumn{3}{|c|}{$\begin{array}{l}\text { Participantes de um Programa de Exer- } \\
\text { cício Físico } \\
\text { (PPEF) }\end{array}$} & \multicolumn{3}{|c|}{$\begin{array}{l}\text { Não Participantes de um Programa de } \\
\text { Exercício Físico } \\
\text { (NPPEF) }\end{array}$} \\
\hline & & $\mathbf{N}$ & & $\%$ & $\mathbf{N}$ & & $\%$ \\
\hline \multirow{2}{*}{ Sexo } & Masculino & 6 & 11,4 & & 6 & 11,4 & \\
\hline & Feminino & 39 & 88,6 & & 39 & 88,6 & \\
\hline \multirow{4}{*}{ Faixa Etária } & 40-49 anos & 5 & 11,1 & & 5 & 11,1 & \\
\hline & 50-59 anos & 13 & 28,9 & & 12 & 26,7 & \\
\hline & 60-69 anos & 21 & 46,6 & & 24 & 53,3 & \\
\hline & $=70$ anos & 6 & 13,4 & & 4 & 8,9 & \\
\hline
\end{tabular}

Nota: Elaboração própria

Os dados apresentados na tabela 1 demonstram que a idade dos componentes dos grupos variou de 61,2 anos para os PPAF, com um desvio padrão de 9,0 anos, para 59,8 para os NPPAF, com desvio padrão de 7,9 anos. Com relação ao gênero, os dois grupos apresentam um maior número de mulheres na sua composição, com $88,6 \%$ da amostra para ambos os grupos. No que se refere à faixa etária, nota-se que o maior quantitativo de pessoas concentra-se no intervalo de 50 a 69 anos, representando $75,5 \%$ do grupo de PPAF e $80 \%$ do grupo NPPAF. 
Tabela 2. Distribuição dos escores de cada domínio avaliado com o questionário SF-36, entre os indivíduos dos grupos PPEF e NPPEF, aplicado em Unidades Básicas de Saúde da Família de Campo Grande (MS) (n=90)

\begin{tabular}{|c|c|c|c|c|c|c|c|c|}
\hline \multicolumn{9}{|c|}{ Distribuição dos escores } \\
\hline \multirow[t]{2}{*}{ Domínios } & \multicolumn{2}{|c|}{ Muito baixo } & \multicolumn{2}{|c|}{ Baixo } & \multicolumn{2}{|c|}{ Elevado } & \multicolumn{2}{|c|}{ Muito elevado } \\
\hline & PPEF & NPPEF & PPEF & NPPEF & PPEF & NPPEF & PPEF & NPPEF \\
\hline $\begin{array}{l}\text { Capacidade } \\
\text { Funcional }\end{array}$ & $2,2 \%$ & $6,7 \%$ & $0,0 \%$ & $17,8 \%$ & $40,0 \%$ & $46,7 \%$ & $57,8 \%$ & $28,9 \%$ \\
\hline Aspectos Físicos & $15,6 \%$ & $57,8 \%$ & $2,2 \%$ & $6,7 \%$ & $2,2 \%$ & $4,4 \%$ & $80,0 \%$ & $31,1 \%$ \\
\hline Dor & $6,7 \%$ & $24,4 \%$ & $13,3 \%$ & $35,6 \%$ & $46,7 \%$ & $22,2 \%$ & $33,3 \%$ & $17,8 \%$ \\
\hline Saúde Geral & $0,0 \%$ & $8,9 \%$ & $17,8 \%$ & $40,0 \%$ & $48,9 \%$ & $42,2 \%$ & $33,3 \%$ & $8,9 \%$ \\
\hline Vitalidade & $0,0 \%$ & $4,4 \%$ & $8,9 \%$ & $28,9 \%$ & $71,1 \%$ & $62,2 \%$ & $20,0 \%$ & $4,4 \%$ \\
\hline Aspectos Sociais & $0,0 \%$ & $4,4 \%$ & $6,7 \%$ & $22,2 \%$ & $22,2 \%$ & $17,8 \%$ & $71,1 \%$ & $55,6 \%$ \\
\hline $\begin{array}{l}\text { Aspectos Emo- } \\
\text { cionais }\end{array}$ & $17,8 \%$ & $53,3 \%$ & $11,1 \%$ & $0,0 \%$ & $2,2 \%$ & $2,2 \%$ & $68,9 \%$ & $44,4 \%$ \\
\hline Saúde Mental & $2,2 \%$ & $8,9 \%$ & $11,1 \%$ & $15,6 \%$ & $37,8 \%$ & $37,8 \%$ & $48,9 \%$ & $37,8 \%$ \\
\hline
\end{tabular}

Nota: Elaboração própria

Considerando os oito domínios previstos no SF-36 e os escores referentes ao mesmo, a tabela 2 demonstra a concentração relativa aos componentes dos grupos dispostos para este estudo, sendo que os resultados obtidos nos permitem afirmar que, quando comparam-se os escores situados nas extremidades 'muito baixo' e 'muito elevado', os dados revelam condições opostas entre os grupos investigados. O escore 'muito baixo' apresenta menor concentração de pessoas pertencentes ao grupo PPAF na maioria dos domínios, enquanto o escore 'muito elevado' demonstra que os integrantes do grupo NPPAF estão em menor quantidade, quando comparado ao outro grupo. A concentração relativa de PPAF apresenta-se mais próxima dos escores/domínios relacionados a uma melhor qualidade de vida, quando comparado com o grupo NPPAF.

Dessa forma, é possível compreender que a prática de atividade física pode influenciar o aumento da qualidade de vida das pessoas, ao contrário daquelas que não a praticam. Tal condição atende a um dos objetivos da Portaria n. ${ }^{\circ}$ 154, aprovada em 2008, que cria os Núcleos de Apoio à Saúde da Família (Nasf) e prevê a prática de atividade física como uma de suas ações, considerando que ela deve propiciar melhoria da qualidade de vida da população, entre outros.

Os resultados apresentados neste estudo corroboram pesquisas prévias que demonstram a influência da atividade física na qualidade de vida das pessoas (MOTA ET AL., 2006; GONÇALVES ET AL., 2011; TOSCANO ; OLIVEIRA, 2009; SILVA ET AL., 2010). 


\begin{tabular}{|c|c|c|c|c|c|}
\hline \multirow{2}{*}{ Domínios } & \multirow{2}{*}{ Grupo } & \multicolumn{4}{|c|}{ Distribuição dos escores } \\
\hline & & Mínimo & Mediana & Máximo & Valor de $p$ \\
\hline \multirow{2}{*}{ Capacidade Funcional } & PPEF & 20 & 80 & 100 & \multirow{2}{*}{$0,0003^{*}$} \\
\hline & NPPEF & 15 & 55 & 95 & \\
\hline \multirow{2}{*}{ Aspectos Físicos } & PPEF & 00 & 100 & 100 & \multirow{2}{*}{0,0001 * } \\
\hline & NPPEF & 00 & 00 & 100 & \\
\hline \multirow{2}{*}{ Dor } & PPEF & 10 & 64 & 100 & \multirow{2}{*}{0,0001 * } \\
\hline & NPPEF & 00 & 41 & 100 & \\
\hline \multirow{2}{*}{ Saúde Geral } & PPEF & 25 & 67 & 100 & \multirow{2}{*}{0,0003 * } \\
\hline & NPPEF & 00 & 51 & 77 & \\
\hline \multirow{2}{*}{ Vitalidade } & PPEF & 25 & 60 & 95 & \multirow{2}{*}{0,0164 * } \\
\hline & NPPEF & 20 & 55 & 75 & \\
\hline \multirow{2}{*}{ Aspectos Sociais } & PPEF & 25 & 87,5 & 100 & \multirow{2}{*}{0,0435 * } \\
\hline & NPPEF & 12,5 & 75 & 100 & \\
\hline \multirow{2}{*}{ Aspectos Emocionais } & PPEF & 00 & 100 & 100 & \multirow{2}{*}{0,0196 * } \\
\hline & NPPEF & 00 & 00 & 100 & \\
\hline \multirow{2}{*}{ Saúde Mental } & PPEF & 12 & 72 & 100 & \multirow{2}{*}{0,0743} \\
\hline & NPPEF & 00 & 68 & 100 & \\
\hline
\end{tabular}

Notas: Elaboração própria

* valores de um mesmo domínio diferem estatisticamente entre si pelo teste de Mann-Whitney $(p<0,05)$

Analisando as variáveis apresentadas (tabela 3), os dados nos permitem afirmar que foram obtidas diferenças estatisticamente significativas na maior parte dos domínios entre os grupos investigados (PPAF e NPPAF), indicando uma associação positiva entre atividade física e qualidade de vida. No que se refere ao domínio 'Saúde Mental', percebe-se que, apesar de o referido domínio não apresentar diferença significativa entre os grupos investigados, ambos se encontram com valores altos em suas pontuações, estando mais próximos de 100, o que, de acordo com o SF-36, indica uma melhor qualidade de vida, do que o valor 0 , que corresponde a uma pior qualidade de vida.

Nossos achados concordam com o estudo de revisão de literatura sistematizada realizado por Pucci et al. (2012), objetivando identificar a associação entre atividade física e qualidade de vida em adultos, o qual verificou que as pesquisas levantadas apontaram relação positiva entre a prática da atividade física e os domínios 'Função Física', 'Vitalidade', 'Papel Físico', 'Papel Emocional', 'Saúde Geral' e 'Saúde Mental'. Os autores avaliaram trinta e oito estudos, sendo o SF-36 o questionário mais utilizado, entretanto, como os instrumentos utilizados foram distintos, incluíram domínios da qualidade de vida diferentes.

Não obstante as conclusões apontadas no estudo de Pucci et al. (2012), as amostras populacionais investigadas foram heterogêneas, sendo os estudos compostos por grupos de idosos, institucionalizados ou não, adultos em programas experimentais de atividade física, adultos em atividade física durante o tempo de lazer, atividade física em pacientes com câncer, adultos em atividade física no 
seu transporte, com grupo de mulheres com sobrepeso, grupo de adultos em reabilitação cardiovascular, cardiopatas, entre outros.

A participação em programas comunitários de atividade física, especificamente para usuários do SUS, pode, conforme Putzel et al. (2011), melhorar, entre outros aspectos, sua qualidade de vida. Em sua pesquisa, os autores supracitados identificaram que os sujeitos investigados perceberam melhoria na condição física, na socialização, na realização das Atividades de Vida Diária (AVD), diminuição do estresse, provocando perda de peso e melhoria da qualidade de vida.

A relação complexa entre saúde e seus determinantes, no Brasil, impõe o desafio de encontrar mecanismos para o enfrentamento das Doenças e Agravos Não Transmissíveis (Dant). No Brasil, as Dant e as causas externas foram as principais causas de morte em 2009, correspondendo a 85,0\% do total de óbitos. Nesse cenário, o Ministério da Saúde propõe, juntamente com outras instituições, o Plano de Ações Estratégicas para o enfrentamento das Dant no Brasil (2011-2012), no qual aborda quatro fatores de risco modificáveis, entre eles, a inatividade física (BRASIL, 2012). Tal proposta converge com a implantação e o apoio aos programas de atividade física vinculados às políticas públicas (BRASIL, 2007), como a aprovação da Política Nacional de Promoção da Saúde, de 2006.

As propostas supracitadas induzem à criação de programas comunitários de atividade física em unidades de saúde, com supervisão profissional, que permitem ao usuário a modificação do estilo de vida de sedentário para ativo, o que, de acordo com Kokubun et al. (2007), deve ocorrer por meio do acesso a programas de atividade física de qualidade. Tais programas, na perspectiva de Simões et al. (2009), são benéficos para a adoção do estilo de vida ativo e contribuem para o aumento dos níveis de atividade física e de lazer.

\section{Considerações finais}

A descrição dos elementos que influenciam a qualidade de vida da população atendida em unidades de saúde vinculadas ao SUS é importante para a tomada de decisão dos gestores, uma vez que subsidiam tecnicamente esses profissionais, para a definição de ampliação, manutenção ou redução de investimentos em determinadas áreas de intervenção. No caso do presente estudo, há evidências de que o investimento no oferecimento de programas de atividade física no SUS pode melhorar as condições de vida da população que busca atendimento na atenção primária.

Na realização desta pesquisa, verificou-se que as UBSF podem tornar-se locais privilegiados para a promoção da saúde e da qualidade de vida de seus usuários, proporcionando a esses o acesso a programas de atividade física. Considera-se, também, a possibilidade de estruturação desses programas no espaço das UBSF, em perspectiva mais ampla, onde, além da atividade física em si, esses possam ser acompanhados de ações de aconselhamento, intersetorialidade, monitoramento e avaliação, conforme previsto nas Políticas Públicas de Promoção à Saúde, na medida em que é previsto que as ações desenvolvidas em tal espaço devem ser subsidiadas por essa perspectiva teórica.

Considera-se que estudos envolvendo essa temática, com a especificidade da população investigada, devam ser replicados, pois, de acordo com a revisão de literatura deste estudo, constatou-se que pesquisas envolvendo atividade física e qualidade de vida nessa população ainda são bastante escassas. 


\section{Referências}

ARAÚJO, D. S. M. S.; ARAÚJO, C. G. S. Aptidão física, saúde e qualidade de vida relacionada à saúde em adultos. Rev. Bras. Med. Esporte, Niterói, v. 6, n. 5, p. 194 203, set./out. 2000.

BLAIR, S. N. et al. Physical activity, physical fitness, and all-cause and cancer mortality: a prospective study of men and women. Ann Epidemiol., New York, v. 6, n. 5, p. 452-7, 1996.

BRASIL. Ministério da Saúde. Portaria GM/MS n ${ }^{\circ}$ 399, de 22 de fevereiro de 2006. Divulga o pacto pela saúde 2006 - consolidação do SUS e aprova as diretrizes operacionais do referido pacto. 2006. Diário Oficial [da] União. Brasília, DF. 22 fev. 2006. Disponível em: <http://dtr2001.saude.gov.br/sas/PORTARIAS/ Port2006/GM/GM-399.htm>. Acesso em: 13 ago. 2014.

Ministério da saúde. Secretaria de Vigilância em Saúde. Departamento de Análise de Situação de Saúde. Plano de ações estratégicas para o enfrentamento de Doenças Crônicas não Transmissíveis no Brasil, 2011-2012. Brasília, DF: Ministério da Saúde, 2012. Disponível em: <http://portal.saude.gov.br/portal/ saude/profissional/area.cfm?id_area $=1818 \geq$. Acesso em: 10 jun. 2013.

Ministério da Saúde. Secretaria de Vigilância em Saúde. Secretaria de Atenção à Saúde. Política Nacional de Promoção da Saúde. 2 ed. Brasília, DF: Ministério da Saúde, Secretaria de Vigilância em Saúde, Secretaria de Atenção à Saúde 2006, 51 p. (Série B Textos básicos de saúde).

CASTELLÓN, A.; PINO, S. Calidad de vida em la atención al mayor. Revista Multidisciplinar de Gerontologia, Barcelona, v. 13, n. 3, jul./ago., 2003, p. 188-192.

CICONELLI, R. M. et al. Tradução para a língua portuguesa e validação do questionário genérico de avaliação de qualidade de vida SF-36 (Brasil SF-36). Rev. Bras. de Reumatologia, São Paulo, v. 39, n.3, jan./fev. 1999, p. 143-150.

COUTINHO, S. S. Atividade fisica no Programa de Saúde da Família, em municípios da $5^{\text {a }}$ Regional de Saúde do Estado do Paraná - Brasil. 2005. 141f. Dissertação (Mestrado em Enfermagem em Saúde Pública) - Universidade de São Paulo, Universidade do Centro-Oeste do Paraná, Ribeirão Preto, 2005.
DEVER, G. E. A. A epidemiologia na administração dos serviços de saúde. São Paulo: Pioneira, 1988.

KREJCIE, R. V.; MORGAN, D. W. Determining sample size for research activities. educacional and psichological measurement, Santa Barbara, n. 30, p. 607-610, 1970.

FORATTINI, O. P. Ecologia, epidemiologia e sociedade. São Paulo: Artes Médicas - USP, 1992.

GONÇALVES, A. K. et al. Qualidade de vida relacionada à saúde de adultos entre 50 e 80 anos praticantes de atividade física regular: aplicação do SF-36. Estudos Interdisciplinares sobre Envelhecimento, Porto Alegre, v. 16, especial, 2011, p. 407-420.

IGLESIAS, R. B. Qualidade de vida de alunos trabalhadores que cursam a graduação em enfermagem. $2002.97 \mathrm{f}$. Dissertação (Mestrado em Enfermagem) - Universidade de São Paulo, Escola de Enfermagem, 2002.

KAYSER, B. et al. Caracterização de idosos participantes de programas de atividade física regular. Rev. Ciênc. Méd. Biol., Salvador, v. 11, n. 3, p. 317-321, set./dez. 2012.

KOKUBUN. E. et al. Programa de atividade física em Unidades Básicas de Saúde: relato de experiência no município de Rio Claro-SP. Revista Brasileira de Atividade Física \&t Saúde, Pelotas, v. 12, n. 1, 2007, p. 45-53.

LANDEIRO, G. M. B. et al. Revisão sistemática dos estudos sobre qualidade de vida indexados na base de dados Scielo. Ciência \&t Saúde Coletiva, Rio de Janeiro, v. 16, n. 10, p. 4257-4266, 2011.

MALTA, D. C. et al. A política nacional de promoção da saúde e a agenda da atividade física no contexto do SUS. Epidemiol. Serv. Saúde, Brasília, DF, v. 18. n. 1. mar. 2009, p. 79-86.

MILES, L. Physical activity and health. Nutrition Bulletin, n. 32, p. 314-363, 2007. Disponível em: $<$ http://onlinelibrary.wiley.com/doi/10.1111/j.14673010.2007.00668.x/abstract>. Acesso em: 15 de ago. 2014.

MONTEIRO, R. et al. Qualidade de vida em foco. Revista Brasileira de Cirurgia Cardiovascular, São Paulo, v. 25 , n. 4 , p. 568-574, out./dez. 2010.

MOTA, J. et al. Atividade física e qualidade de vida 
associada à saúde em idosos participantes e não participantes em programas regulares de atividade física. Rev. bras. educ. fís. esporte, São Paulo, v. 20, n. 3, p. 219252, jul./set. 2006.

NAHAS, M. V. Atividade Física, saúde e qualidade de vida: conceitos e sugestões para um estilo de vida ativo. 6 ed. Londrina: Midiograf, 2013.

PAFFENBARGER JR., R. S.; LEE, I. M. Physical activity and fitness for health and longevity. Research Quarterly for Exercise and Sport, Dallas, v. 67, supl. 3, 1996, p. 11-28.

PATE, R. R. et al. Physical activity and public health. A recommendation from the Centers for Disease Control and Prevention and the American College of Sports Medicine. JAMA, Chicago, v. 273, n. 5, p. 402-7, 1995.

PUTZEL, M. P. Efeitos da atividade física no SUS: percepções de usuários e profissionais da saúde. 2011. 70 f. Trabalho de Conclusão de Curso (Especialização em Educação Física) - Universidade Comunitária da Região de Chapecó, Chapecó, 2011.

PUCCI, G. C. M. F. et al. Associação entre atividade física e qualidade de vida em adultos. Rev. Saúde Pública, São Paulo,v. 46, n. 1, p. 166-79, fev. 2012.

SILVA, R. S. et al. Atividade física e qualidade de vida. Ciência \&t saúde coletiva, Rio de Janeiro, v. 15, n. 1, jan. 2010, p. 115-120.
SIMOES, E. J. et al. Effects of a community-based, professionally supervised intervention on physical activity levels among residents of Recife, Brazil. Am J Public

Health, New York, v. 99, n.1, p. 68-75, jan. 2009.

TAIROVA, O. S.; DI LORENZI, D. R. S. Influência do exercício físico na qualidade de vida de mulheres na pós-menopausa: um estudo caso-controle. Revista Brasileira de Geriatria e Gerontologia. Rio de Janeiro, v. 14, n. 1, 2011, p. 135-145.

TOSCANO, J. J. O.;OLIVEIRA, A. C. C. Qualidade de vida em idosos com distintos níveis de atividade física. Rev Bras Med Esporte, Niterói, v. 15, n. 3, p. 169-173, maio/jun. 2009.

WORLD HEALTH ORGANIZATION (WHO). Physical activity: direct and indirect health benefits. Disponivel em: <www.who.int/hpr/physactic/health.benefits.shtml>.

Acesso em: 3 out. 2004.

\footnotetext{
Recebido para publicação em fevereiro de 2015

Versão final em março de 2015

Conflito de interesse: inexistente

Suporte financeiro: não houve
} 